\title{
Angular responses of the first diffracted order in over-modulated volume diffraction gratings
}

\author{
C. Neipp ${ }^{1}$, M. L. Alvarez ${ }^{1}$, S. Gallego ${ }^{2}$, M. Ortuño ${ }^{2}$, J. Sheridan ${ }^{3}$, \\ I. Pascual ${ }^{2}$ and A. Beléndez ${ }^{1}$ \\ ${ }^{1}$ Departamento de Física, Ingeniería de Sistemas y Teoría de la Señal, Universidad de \\ Alicante, Ap. 99, E-03080 Alicanfe, Spain \\ ${ }^{2}$ Departamento Interuniversitariơo de Óptica, \\ Universidad de Alicante, Apartado99, E-03080 Alicante, Spain \\ ${ }^{3}$ Departament of Electronic and Electrical Engineering, University College Dublin, \\ Belfield, Dublin 4, Republic of Ireland \\ Tel.: +34-6-5903682 \\ Fax: +34-6-5909750 \\ E-mail: cristian@disc.ua.es
}




\begin{abstract}
Kogelnik's Coupled Wave Theory has been used for decades to predict the diffraetion efficiency of volume diffraction gratings. Although this theory has been applied with success to volume diffraction gratings recorded under a great variety of experimental conditions, its predictions deviate from the actual behaviour whenever the hologram is thin or the refractive index is high. In these cases, it is necessary to use a more general Coupled Wave Theory (CW) or the Rigorous Coupled Waye Theory (RCW). Both of these theories allow for more than two orders propagating inside the hologram. The difference between them is that in the $\mathrm{CW}$ theorythe second derivatives that appear in the coupled equations are disregarded. The RCW doesn't incorporate any approximation and thus, since it is rigorous, permits judging the accuracy of the approximations included in Kogelnik's and CW theories. In this article a comparison between the predictions of the three theories for phase transmission diffraction gratings is carried put. Over-modulated diffraction gratings are also recorded in photographic emulsions in order to study the applicability of Kogelnik's Theory in this case. Good agreement between theory and experiment is found for both Kogelnik's and Rigorous Coupled Wave Theory formulations in the particular experimental cases studied.
\end{abstract}

Keywords: Holography; Volume holograms, Holographic recording materials, Photographic emulsions. 


\section{Introduction}

Volume phase holograms are of relatively high importance due to their applications in different fields. In particular, a lot of research has been done in the field of holographic phase gratings. Therefore, it is interesting to understand how light propagates inside a periodic medium. And since the beginnings of Holography a lot of research has been done in order to provide an accurate background of theories that could predict the exact behaviour of light inside holograms. The modal theory, first proposed by Wagner [1] and Tamir et al. [2-3] for dielectric gratings, was introduced into holography by Burkhardt [4]. This theory treats the problem of the propagation of light inside the hologram in terms of a set of characteristic modes propagating through the periodic medium. On the other hand, the coupled wave theory (a goód review of it can be found in refs 5-6) assumes that different plane waves, orders, propagate inside the hologram. Through the thickness of the hologram transfer of ênergy occurs between the different orders, thus at the end of the periodic structure, some of them are reinforced with respect to the others. In particular, a two wave coupled wave theory is very popular: that proposed by Kogelnik [7]. Kogelnik's Coupled Wave Theory has the advantage over other theories that, in spite of being mathematically simple, it predicts very accurately the response of the efficiency of the first and second order for volume phase gratings. Nonetheless, the accuracy decreases when either the thickness is low or when over-modulated patterns (high refractive index modulations) are recorded in the hologram. In these cases, the coupled wave theory (CW) allowing for more than two orders or the rigorous 'coupled wave theory (RCW) [8] which doesn't disregard second derivatives in the coupled wave equations as does $\mathrm{CW}$, are needed. As has been demonstrated during the last two decades, since its first introduction by Moharam and Gaylord [8] the RCW method has accomplished the task of explaining a great number of physical situations associated with diffraction gratings of different kinds [9-14]. This theory is also useful to check into what extent the approximations made in other theories are valid.

It has been demonstrated by Moharam et al. [15-17] that the regime of application of Kogelnik's Theory, usually called the Bragg regime, can be found by comparing two parameters, the volume factor, $Q$, and the grating strength, $v$. The volume factor is proportional to the ratio of the thickness, $d$, of the grating to the square 
of the period of the grating, $\Lambda$, whereas the grating strength, controls the diffraction efficiency, $\eta$, at Bragg condition $(\eta=100 \%$ if $v=\pi / 2)$. Two different curves in the $Q$ $v$, plane, $v Q=$ const, $Q / v=$ const, delimit the Raman-Nath and Bragg regimes. Although, several theoretical simulations have been performed to compare the different coupled wave theories, sometimes these simulations make use of physical values which are not practical in holography. Therefore, it is interesting to check the range of applicability of these theories when the parameters take physically obtainable values. In this work we will study the applicability of Kogelnik's Coupled Wave Theory to different situations. To do this the efficiency response of the first order at finst Bragg angle condition for transmission gratings with different spatial frequencies will be investigated. In this study, two different refractive index modulations will also be used: a relatively easily achievable one in many materials, such as photographic emulsions, dichromated gelatins or silver halide sensitised gelatins, $n_{1} \neq 0.025$, and a relatively high one, which will allow us to study the efficiency of the first order in over-modulated patterns, $n_{l}=0.055$.

\section{Efficiency of the different orders that propagate in the diffraction grating}

\subsection{Coupled wave equations}

In this section the differential equations that govern the behaviour of the different diffracted orders propagating inside a sinusoidal transmission diffraction grating will be dêrived. Since these equations are well known [5-6] only a brief description is given here.

We will study the propagation of light inside a phase sinusoidal transmission grating. For simplicity the conductivity inside the grating, $\sigma$, is supposed to be zero and the reelative permittivity in the hologram is expressed as:

$$
\varepsilon_{r}(x, z)=\varepsilon_{r 0}+\varepsilon_{r 1} \cos \boldsymbol{K} \cdot \boldsymbol{r}
$$

where $\varepsilon_{\mathrm{r} 0}$ is the average dielectric constant, $\varepsilon_{\mathrm{r} 1}$ the amplitude of the relative permittivity and $\boldsymbol{K}$ is the grating vector, which is related to the period of the interference fringes, $\Lambda$, as follows: 


$$
|\boldsymbol{K}|=\frac{2 \pi}{\Lambda}
$$

Following the $\mathrm{CW}$ and RCW approaches the electric field inside the hologram is supposed to be an infinite sum of orders in the form:

$$
E_{1}=\sum_{i} S_{i}(z) \exp \left(-j \rho_{i} \cdot \boldsymbol{r}\right) \quad \mathrm{i}=0, \pm 1, \pm 2, \ldots
$$

where $S_{\mathrm{i}}$ and $\rho_{\mathrm{i}}$ are the amplitude and the propagation vector of the $\mathrm{i}^{\text {th }}$ diffracted order, respectively. The propagation vector is related to the grating vector as:

$$
\boldsymbol{\rho}_{\mathrm{i}}=\boldsymbol{\rho}_{0}+i \boldsymbol{K}
$$

$\boldsymbol{\rho}_{0}$ being the propagation vector of the incident wave.

Since the electric vector $E_{1}$ satisfies the wave equation insidethe hologram:

$$
\nabla^{2} E_{1}+\beta^{2}\left(1+\frac{\varepsilon_{r 1}}{\varepsilon_{r 0}} \cos \boldsymbol{K} \cdot \boldsymbol{r}\right) E_{1} \in \mathcal{O}_{9}
$$

$\beta$ is the propagation constant inside the hologram,

$$
\beta=\frac{2 \pi}{\lambda}\left(\varepsilon_{r 0}\right)^{1 / 2}
$$

and $\lambda$ is the wavelength of light in vacuum.

By substituting equations (1) and (3) into (5) the following well-known equations are obtained:

$$
\frac{d^{2} S_{i}(z)}{d z^{2}}-2 j \rho_{i z z} \frac{d S_{i}(z)}{d z}+\left(\beta^{2}-\left|\rho_{i}\right|^{2}\right) S_{i}(z)+\left(\beta^{2} \cdot \frac{\varepsilon_{r 1}}{2 \varepsilon_{r 0}}\right)\left(S_{i+1}(z)+S_{i-1}(z)\right)=0
$$

This set of differential equations govern the behaviour of the several diffracted orders propagating inside the periodic medium. It is usually interesting to express (7) as:

$$
\frac{j}{2 \beta} \frac{d^{2} S_{i}(z)}{d z^{2}}+C_{i} \frac{d S_{i}(z)}{d z}-j \kappa \Omega i(i+P) S_{i}(z)+j \kappa\left(S_{i+1}(z)+S_{i-1}(z)\right)=0
$$

$C_{\mathrm{i}}$ are the called obliquity factors and are the cosine of the angles that the propagation vectors of the different orders form with the $\mathrm{z}$ axis. In the particular case of non-slanted diffraction gratings, $C_{\mathrm{i}}=\cos \theta_{0}$, where $\theta_{0}$ is the angle of reconstruction inside the medium. 
The parameter $\kappa$ is the coupling constant and is defined as:

$$
\kappa=\frac{\beta \varepsilon_{r 1}}{4 \varepsilon_{r 0}}
$$

The $\Omega$ parameter is defined as:

$$
\Omega=\frac{|\boldsymbol{K}|^{2}}{2 \beta \kappa}
$$

As has been demonstrated by Solymar and Cooke [5], $\Omega$ is a parameter which gives a criterion of whether the hologram is thin or thick. It is stated in ref. 5 that whenever $\Omega>5$ the hologram can be considered thick.

Finally, the parameter $P$ is defined as:

$$
P=\frac{2 \beta}{|\boldsymbol{K}|} \sin \left(\theta_{0}-\varphi\right)
$$

$\varphi$ is the angle between the fringes and the $\mathrm{z}$ axis, which for non-slanted geometry is 0 . The parameter $P$ is the called impact parameter and takes the values, $P=1$ for reconstruction at first Bragg angle, $P=2$ at the second Bragg angle, and so on. In this work we shall restrict the study to the first Bragg condition.

It has been a common strategy in volume Holography to disregard the second derivatives of equation (8), since slow variation of the diffracted orders inside the grating is supposed. This is not completely true for high values of the coupling constant, $\kappa$, but as will be demonstrated in this study in the range of values that will be treated here, this approximation is perfectly valid.

If the second derivatives are disregarded, equation (8) is transformed into the following, which is easier to handle:

$$
C_{i} \frac{d S_{i}(z)}{d z}-j \kappa \Omega i(i+P) S_{i}(z)+j \kappa\left(S_{i+1}(z)+S_{i-1}(z)\right)=0
$$

or in order to reduce the amount of significant parameters:

$$
\frac{d S_{i}(z)}{d \zeta}-j \Omega i(i+P) S_{i}(\zeta)+j\left(S_{i+1}(\zeta)+S_{i-1}(\zeta)\right)=0
$$

where: 


$$
\zeta=\frac{\kappa z}{\cos \theta_{0}}
$$

Now, equation (13) has only two significant parameters, $\Omega$ and $P$. The influence of $\Omega$ and $P$ can easily be interpreted from equation (13). Coupling from one order to the two adjacent ones takes place through the last term of this equation. The importance of this term grows as $\Omega$ decreases, diminishing the influence of the second term. Therefore multi-wave diffraction occurs whenever $\Omega$ is small. Although this argument can be considered general, the rigorous equation (8) cannot be expressed in terms of only two significant parameters, $\Omega$ and $P$, so a more complex interpretation must be made. Nonetheless, it can be seen that coupling from one order to the adjacent ones is strengthen whenever the value of $\kappa$ increases, and the product $\Omega \kappa$ diminishes. Thus, for high values of $\kappa$ and low values of Q multi-wave diffraction occurs.

\subsection{Solution of the rigorous coupled wave equation}

In this section we will explain the method of solution of equation (8), which is based in a well-known method, first introduced by Moharam and Gaylord [8]. Although the method is applicable to holographic gratings surrounded by two different media, we will suppose that the diffraction grating is embedded in an index-matching medium. The method will also be restricted to unslanted transmission geometry. These assumptions are done in order to make an effective comparison between solutions obtained from equation (8) and (12), under simple conditions.

By using the following change of variables

$$
S_{2, i}=\dot{S}_{1, i}
$$

wherethe upper dot represents the derivative with respect to variable $\mathrm{z}$.

equation (8) can be transformed into:

$$
\dot{S}_{2, i}=a S_{1, i+1}+b_{i} S_{1, i}+a S_{1, i-1}+c_{i} S_{2, i}
$$

where:

$$
\begin{gathered}
a=-2 \beta \kappa \\
b_{\mathrm{i}}=2 \beta \kappa \Omega i(i+P)
\end{gathered}
$$




$$
c_{\mathrm{i}}=2 \beta \cos \theta_{0}
$$

In matrix form equation (16) is converted into:

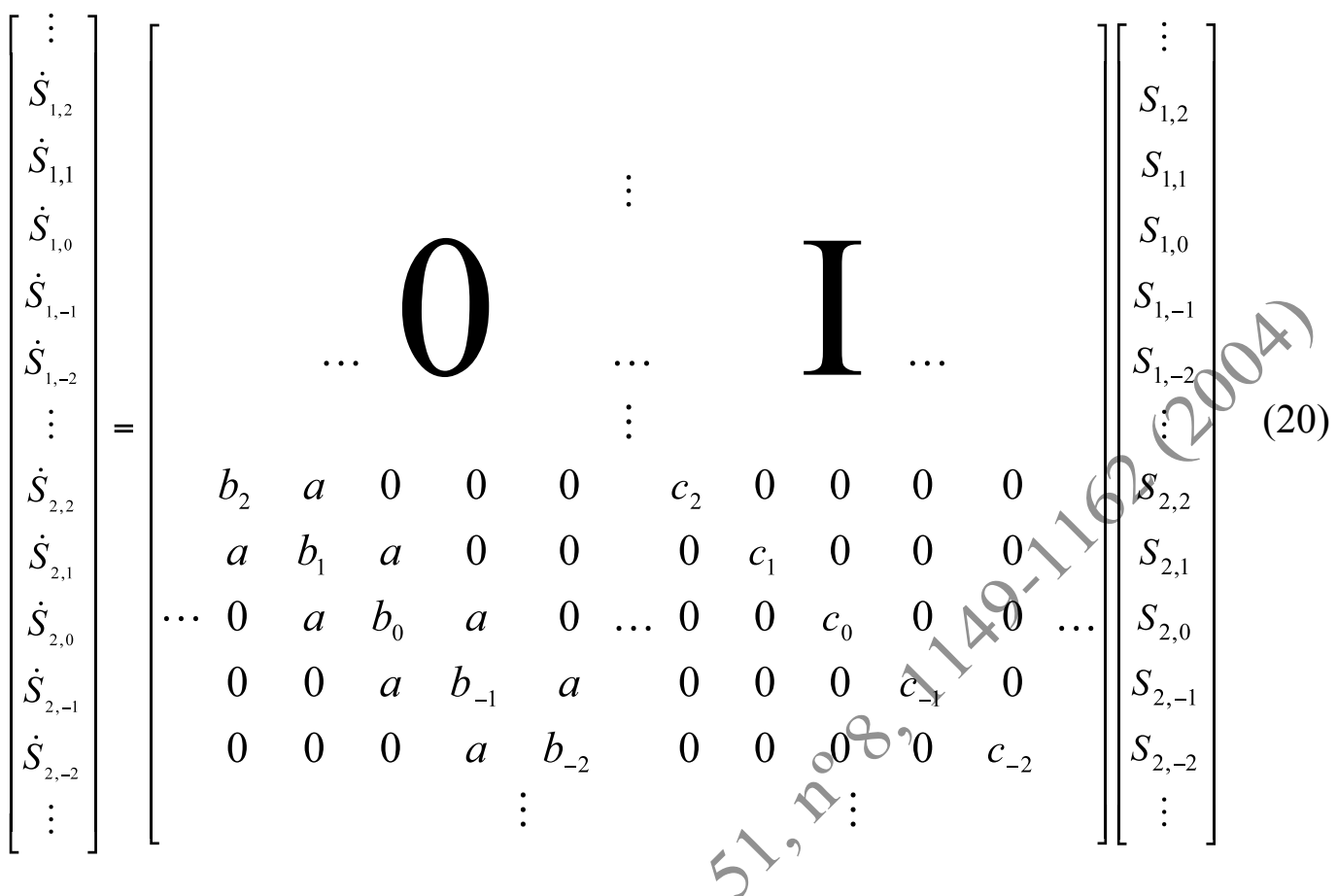

The solution of the amplitude of the different orders can be obtained in terms of the eigenvalues and eigenvectors of the central matrix in equation (20), which we will call A.

$$
S_{i}(z)=\sum_{m} C_{m} w_{i m} \exp \left(q_{m} z\right)
$$

$q_{\mathrm{m}}$ is the $\mathrm{m}^{\text {th }}$ eigenvalue of matrix $\mathbf{A}$, whereas $w_{\text {im }}$ is the $\mathrm{m}^{\text {th }}$ column element of the row corresponding to the $\mathrm{i}_{9}^{\text {th }}$ order in the matrix composed of the eigenvectors of matrix A.

In order to obtain the values of constants $C_{m}$ the adequate boundary conditions must be imposed.

The field inside the periodic medium can be expressed as:

$$
E_{1}=\sum_{i} S_{i}(z) \exp \left\{-j\left[\beta_{i} x+\xi_{i} z\right]\right\}
$$

where in non-slanted geometry:

$$
\xi_{\mathrm{i}}=\beta C_{\mathrm{i}}=\beta \cos \theta
$$

and

$$
\beta_{\mathrm{i}}=\beta \sin \theta+i K
$$


At the output of the hologram the electric field is expressed as:

$$
E_{2}=\sum_{i} T_{i} \exp \left\{-j\left[\beta_{i} x+\xi_{i}(z-d)\right]\right\}
$$

Where $T_{i}$ are the amplitude of the different diffracted orders at the output of the hologram.

To obtain the unknown $C_{m}$ constants two conditions will be imposed:

1) At $z=0$ only the zero order propagates:

$$
S_{i}(0)=\delta_{i 0}
$$

by using (21):

$$
\delta_{i 0}=\sum_{m} C_{m} w_{i m}
$$

2) At $z=d$ the tangential components of the electric and magnetic fields are continuous:

$$
\begin{gathered}
T_{i}=\sum_{m} C_{m} w_{i m} \exp \left[\left(q_{m}-j \xi \vartheta d\right]\right. \\
T_{i}\left(-j \xi_{i}\right)=\sum_{m} C_{m} w_{i m}\left(q_{m}-j \xi_{i}\right) \exp \left[\left(q_{m}-j \xi_{i}\right) d\right]
\end{gathered}
$$

from equations (28) and (29) this other relation can be obtained:

$$
0=\sum_{m} C_{m} w_{i m} q_{m} \exp \left[q_{m} d\right]
$$

Equations (27) and (30) permit obtaining the $2 \mathrm{xN}$ unknown $C_{m}$ coefficients, where $\mathrm{N}$ is the number of orders retained in the calculations. Finally from equation (28) the amplitudes of the $\mathrm{N}$ diffracted orders at the end of the periodic structure can be obtained. The efficiency of the $\mathrm{i}^{\text {th }}$ order is calculated as:

$$
D E_{\mathrm{i}}=T_{\mathrm{i}} T_{\mathrm{i}}^{*}
$$

\subsection{Comparison between $C W, R C W$ and Kogelnik's theory}

It was previously commented that the parameters $\Omega$ and $\kappa$ provide a criterion whether the hologram could be considered thin or thick. In fact, if we restrict our study to the first Bragg condition, $P=1$, and disregard the second derivatives, thus obtaining 
equation (13), the only significant parameter is $\Omega$. Nonetheless in the case of equation (8), or RCW, this study is not so simple.

In this section we will compare the solutions obtained from equations (8) and (13) and the analytical expression obtained by Kogelnik for the efficiency of the first order at first Bragg condition. 9 orders retained in the calculations for equations (8) and (13): $\mathrm{N}= \pm \mathrm{i}, \mathrm{i}=0,1,2, \ldots$ In this study different spatial frequencies will be considered and two values of the refractive index modulation $n_{l}$ will be assumed, a relatively easy achievable value in most usual materials, $n_{1}=0.025$, and a relatively high one, $m_{1}=$ 0.055. Especially interesting is the comparison for the second value, since Kogenik's Theory will be tested in for over-modulated holograms.

It should be noticed that $\mathrm{CW}$ and RCW theories assume a harmonic dependence of the relative permitivity, whereas experimental researchers in Holography usually describe the phase grating in terms of the refractive index. Therefore, it is necessary to relate the average and first harmonic component of the refractive index to the average and first harmonic component of the dielectric permittivity. Using:

$$
\varepsilon_{1}=2 n_{0} n_{1}
$$

where $n_{0}$ is the average refractive index and $n_{1}$ is the refractive index modulation. Equation (32) holds whenever $n_{1}<<n_{0}$. In the theoretical calculations a value of $n_{0}=$ 1.63 was used, which is a practical value for materials such as photographic emulsions.

\section{dInsert Figure 1 about here]}

\section{[Insert Figure 2 about here]}

Figures 1 and 2 show the efficiency of the first order, +1 , as a function of the thickness, $d$, under Bragg condition for a transmission diffraction grating with a spatial frequency of 350 lines $/ \mathrm{mm}$. For the theoretical simulations of Figure 1 a value of $n_{1}=$ 0.025 was considered, whereas for Figure 2 the value of $n_{1}=0.055$ (over-modulated) was assumed. It can be seen that in both cases the theoretical simulations obtained by using the CW and RCW method differ clearly from that obtained by using Kogelnik's Theory. The values of $\Omega$ (from equation (10)) obtained for all the theoretical gratings considered are given in Table 1. The values of $\Omega$ took the values of 1.20 and 0.55 for the gratings considered in figures 1 and 2. For such a low spatial frequency it is clear that multi-wave diffraction occurs and Kogelnik's Theory is not applicable. 
Nonetheless, it is interesting to notice that good agreement between $\mathrm{CW}$ and RCW simulations is obtained, justifying the neglect of the second derivatives in this case.

[Insert Table 1 about here]

[Insert Figure 3 about here]

[Insert Figure 4 about here]

Figures 3 and 4 represent the results of the diffraction efficiency as a function of the thickness for a 500 lines $/ \mathrm{mm}$ spatial frequency diffraction grating. A value of $n_{1}=$ 0.025 was considered in Figure 3 whereas $n_{1}=0.055$ was chosen for the theoretical simulations of Figure 4. In the case of $n_{l}=0.025$ Kogelnik's, CW and RCW Theories all agree, but they disagree in the over-modulated case $\left(n_{1}=0.055, \Omega=1.12\right)$. Again, there are almost no differences between $\mathrm{RCW}$ and $\mathrm{CW}$ theories for both cases.

[Insert Figure 5 about here]

[Insert Figure 6 about here]

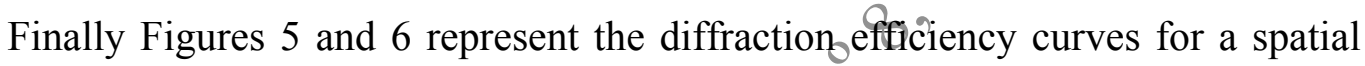
frequency of 750 lines/mm and Figures 7 and 8 for a spatial frequency of 1200 lines/mm. In these cases, even in the over-modulated case, good agreement between the three theories is observed. It is interesting to notice that for the grating analyzed in Figure 6 a value of $\Omega=2.51<5$, is calculated, so the Bragg regime is not guaranteed, nonetheless Kogelnik's Theory seems to be applicable for this case.

[Insert Figure 7 about here]

[Insert Figure 8 about here]

\section{Angular responses of the first diffracted order for over-modulated gratings}

In this section, experimental results of the angular responses of the diffraction efficiency will be shown. The theoretical functions obtained by using the RCW and Kogelnik's Theory will also be compared to the experimental data.

The experiments were carried out with BB-640 plates, an ultra-fined grain red sensitive emulsion. Non-slanted holographic gratings were recorded on BB-640 emulsion by the interference of two collimated beams from a He-Ne laser $(633 \mathrm{~nm})$. The experimental set-up is depicted in Figure 9. The beam ratio was 1:1 and the polarisation plane was normal to the plane of incidence (TE polarisation). The angle between the normal of the plate and each of the incident beams was $22.5^{\circ}$, so that the 
spatial frequency of the diffraction gratings was calculated as $\sim 1200$ lines $/ \mathrm{mm}$. After exposure the holograms underwent a fixation-free rehalogenating bath [18-20] so finally phase volume gratings were obtained. In other works it has been demonstrated that fixation-free rehalogenating bleaching techniques produce phase holograms with high values of the refractive index modulation. Therefore, by using these techniques overmodulated patterns could be obtained.

\section{[Insert Figure 9 about here]}

The diffraction efficiency of the phase holograms was calculated as the ratio of the diffracted beam intensity to the incident power, and Fresnel losses were also taken into account.

\section{[Insert Figure 10 about here]}

[Insert Figure 11 about here]

Figures 10-13 show the results of the diffraction efficiency for volume phase transmission gratings recorded on BB-640 emulsion usingfixation free rehalogenating techniques. The dotted points correspond to the experimental data, the dashed line to the theoretical simulation using Kogelnik, while the continuous line corresponds to the simulation performed using the RCW theory Th all cases high values of the refractive index modulation were recorded. The Figures are presented in order of increasing index modulation. In Table 2 the parameters used in the theoretical simulations are presented for each case. The most important fact that can be deduced from the graphs is that Kogelnik's theory is highly applicable for volume holograms presenting high values of the volume factor, Q, even in high over-modulated cases, such as Figure 13 where a refractive index as high as $n_{l}=0.099$ was stored in the hologram.

\section{[Insert Table 2 about here] \\ [Insert Figure 12 about here] \\ [Insert Figure 13 about here]}

\section{Conclusions}

The limits of applicability of Kogelnik's Theory have been studied for a particular range of physically possible values. It has been demonstrated that for unslanted transmission gratings recorded with spatial frequencies of over 750 lines $/ \mathrm{mm}$, even for high 
refractive index modulations, Kogelnik's Theory is applicable in a range of thickness between $\left[\begin{array}{ll}0 & 18\end{array}\right] \mu \mathrm{m}$. The theoretical simulations also demonstrate that $\mathrm{CW}$ and $\mathrm{RCW}$ theories yield the same results for the first diffracted order at the first Bragg angle replay condition.

\section{Acknowledgements}

This work was supported by the Ministerio de Ciencia y Tecnología, CICYT, Spain, under project MAT2000-1361-C04-04 and by the "Oficina de Ciencia y Tecnologia" (Generalitat Valenciana, Spain) under project GV01-130. 


\section{References}

[1] Wagner, E. H., 1996, Zeitung Physik, 141, 602-621 (I); 622-643 (II).

[2] Tamir, S., Wang, H. C., 1966, Canadian Journal of Physics, 44, 2073-2094 (I); 2461-2494 (II).

[3] Tamir, S., Wang, H. C., and Oliner, A.A., 1964, IEEE Trans. Microwave Theory \& Technology, MTT-12, 323-335.

[4] Burckhardt, C. B., 1966, Journal of the Optical Society of America, 56, 15021509.

[5] Solymar, L., and Cooke, D. J., 1981, Volume Holography and Volume Gratings (Academic, London), p. 79.

[6] Syms, R. R. A., 1990, Practical Volume Holography (Glarendon Press, Oxford).

[7] Kogelnik, H., 1969 Bell Systems Technology Journât, 48, 2909-2947.

[8] Moharam, M. G., and Gaylord, T. K., 1981, Journal of the Optical Society of America, 71, 811-818.

[9] Moharam, M. G., and Gaylord, T. K, ,9983, Journal of the Optical Society of America, 73, 451-455.

[10] Moharam, M. G., and Gaylord, T. K., 1983, Journal of the Optical Society of America, 73, 1105-1112

[11] Gaylord, T. K., and Moharam, M. G., 1985, Proceedings of the IEE, 73, 894937.

[12] Moharam, M. G., and Gaylord, T. K., 1986, Journal of the Optical Society of America $4,3,1780-1796$.

[13] Moharam, M. G., Grann, E. B., Pommet, D. A., and Gaylord, T. K., 1995, Sournal of the Optical Society of America A, 12, 1068-1076.

[14] • Kamiya, N., 1998, Applied Optics, 37, 5843-5853.

[15] Moharam, M. G., and Young, L., 1978, Applied Optics, 17, 1757-1759.

[16] Moharam, M. G., Gaylord, T. K., and Magnusson, R., 1980, Optics Communications, 32, 19-23.

[17] Moharam, M. G., Gaylord, T. K., and Magnusson, R., 1980, Optics Communications, 32, 14-18. 
[18] Bjelkhagen, H. I., 1995, Silver-Halide Recording Materials (Springer Verlag, Berlin).

[19] Hariharan, P., Ramanathan, C.S., and Kaushik, G.S., 1971, Optics Communications, 3, 246-247.

[20] Neipp, C., Pascual, I., and Beléndez, A., 2000, Journal of Modern Optics, 47, 1671-1679. 


\section{Tables}

Table 1. Values of parameter $\Omega$ for transmission diffraction gratings with different spatial frequencies

\begin{tabular}{|l|llll|}
\hline & $\mathrm{f}=350$ lines $/ \mathrm{mm}$ & $\mathrm{f}=500$ lines $/ \mathrm{mm}$ & $\mathrm{f}=500$ lines $/ \mathrm{mm}$ & $\mathrm{f}=1200$ lines $/ \mathrm{mm}$ \\
\hline \hline $\mathrm{n}_{1}=0.025$ & $\Omega=1.20$ & $\Omega=2.46$ & $\Omega=5.53$ & $\Omega=14.16$ \\
\hline $\mathrm{n}_{1}=0.055$ & $\Omega=0.55$ & $\Omega=1.12$ & $\Omega=2.51$ & $\Omega=6.43$ \\
\hline
\end{tabular}

Table 2. Values of parameters $\alpha, n_{1}, d$ for transmission diffraction gratings recorded on BB-640 emulsions

\begin{tabular}{|c|c|c|c|c|}
\hline & Figure 10 & Figure 11 & Figure 12 & Figure 13 \\
\hline$n_{1}$ & 0.050 & 0.058 & 0.077 & 0.099 \\
\hline$d(\mu \mathrm{m})$ & 7.3 & 7.6 & 7.5 & 7.1 \\
\hline$\alpha\left(\mu \mathrm{m}^{-1}\right)$ & 0.018 & 0.0 .029 & 0.032 & 0.035 \\
\hline
\end{tabular}




\section{Figure Captions}

Figure 1.- Efficiency of the first order, at first Bragg angle condition for a transmission diffraction grating of 350 lines $/ \mathrm{mm}$ with a refractive index modulation of $n_{l}=$ 0.025 .

Figure 2.- Efficiency of the first order, at first Bragg angle condition for a transmission diffraction grating of 350 lines $/ \mathrm{mm}$ with a refractive index modulation of $n_{l}=$ 0.055 .

Figure 3.- Efficiency of the first order, at first Bragg angle condition for a transmission diffraction grating of 500 lines $/ \mathrm{mm}$ with a refractive index modulation of $n_{1}=$ 0.025 .

Figure 4.- Efficiency of the first order, at first Bragg angle condition for a transmission diffraction grating of 500 lines $/ \mathrm{mm}$ with a refractive index modulation of $n_{l}=$ 0.055 .

Figure 5.- Efficiency of the first order, at first Bragg angle condition for a transmission diffraction grating of $750 \mathrm{lines} / \mathrm{mm}$ with á refractive index modulation of $n_{1}=$ 0.025 .

Figure 6.- Efficiency of the first order, at first Bragg angle condition for a transmission diffraction grating of 750 lines/mm with a refractive index modulation of $n_{l}=$ 0.055 .

Figure 7.- Efficiency of the first order, at first Bragg angle condition for a transmission diffraction grating of 1200 lines/mm with a refractive index modulation of $n_{1}=$ 0.025 .

Figure 8.- Efficiency of the first order, at first Bragg angle condition for a transmission diffraction grating of 1200 lines $/ \mathrm{mm}$ with a refractive index modulation of $n_{1}=$ 0.055 .

Figure 9.- Experimental set-up

Figure 10.- Efficiency of the first order as a function of the reconstruction angle for a transmission diffraction grating of 1200 lines/mm recorded on BB-640 emulsion and presenting a refractive index modulation of $n_{1}=0.050$

Figure 11.- Efficiency of the first order as a function of the reconstruction angle for a transmission diffraction grating of 1200 lines/mm recorded on BB-640 emulsion and presenting a refractive index modulation of $n_{1}=0.058$ 
Figure 12.- Efficiency of the first order as a function of the reconstruction angle for a transmission diffraction grating of 1200 lines/mm recorded on BB-640 emulsion and presenting a refractive index modulation of $n_{1}=0.077$

Figure 13.- Efficiency of the first order as a function of the reconstruction angle for a transmission diffraction grating of 1200 lines/mm recorded on BB-640 emulsion and presenting a refractive index modulation of $n_{1}=0.099$ 

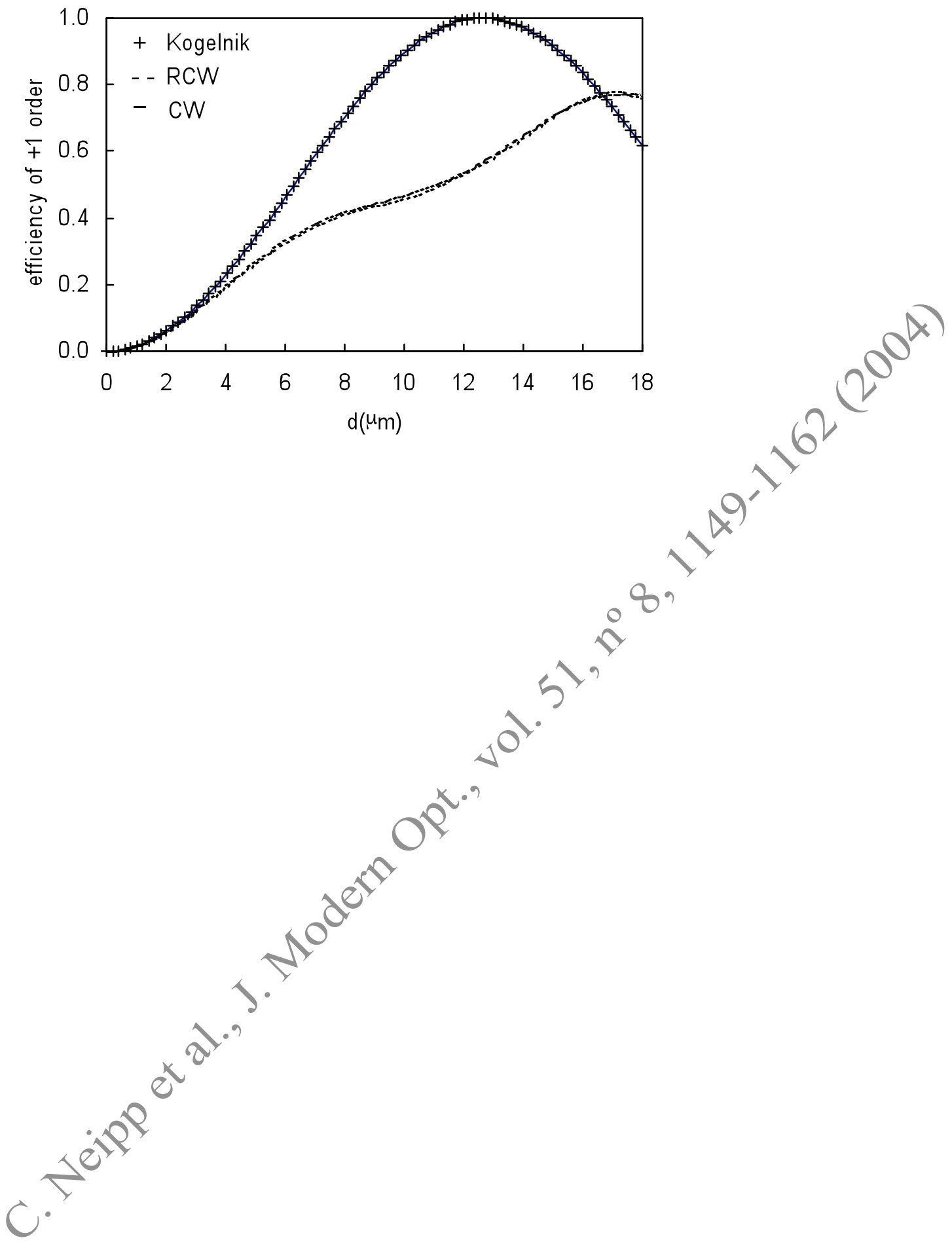

Figure 12 

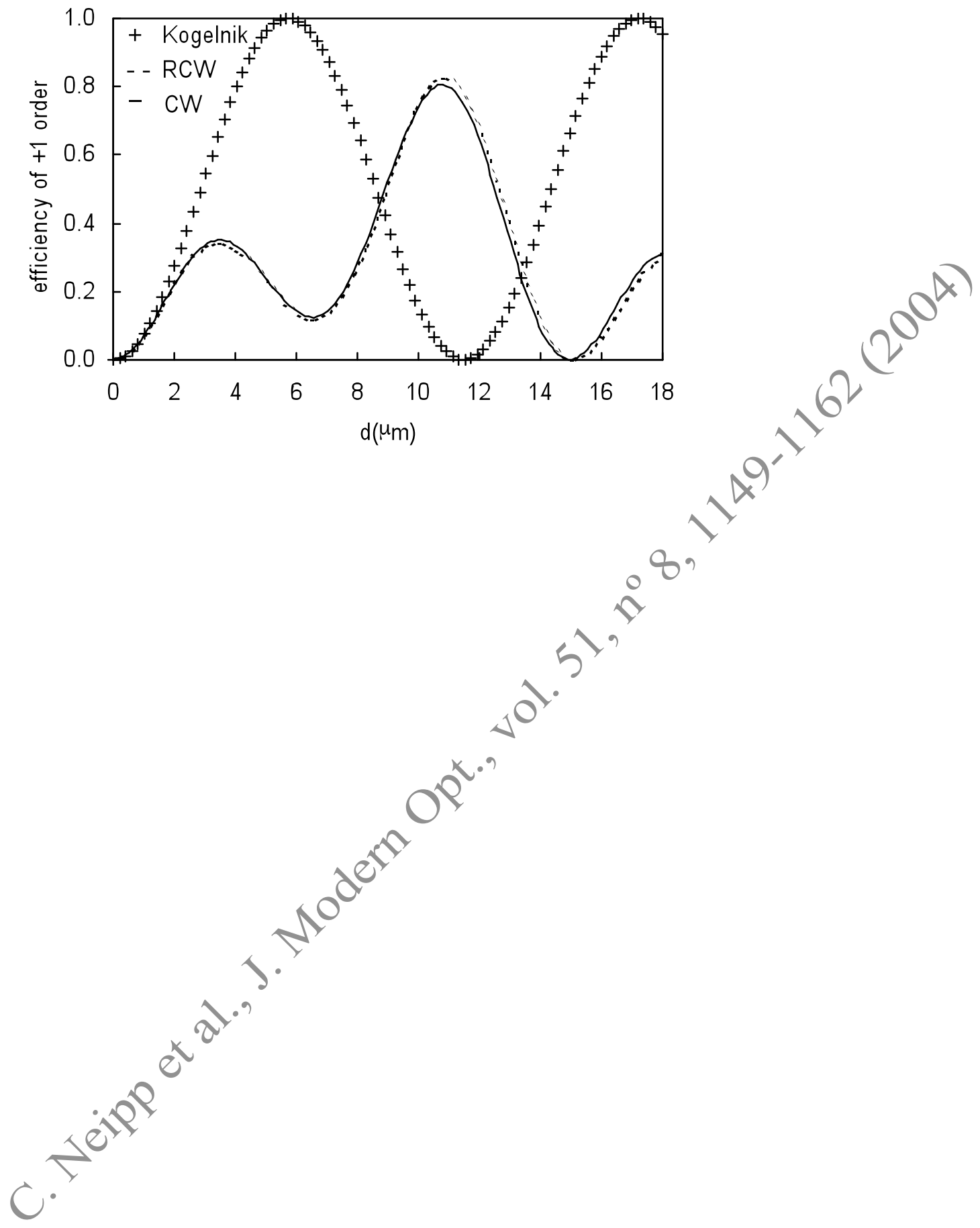

Figure 12 

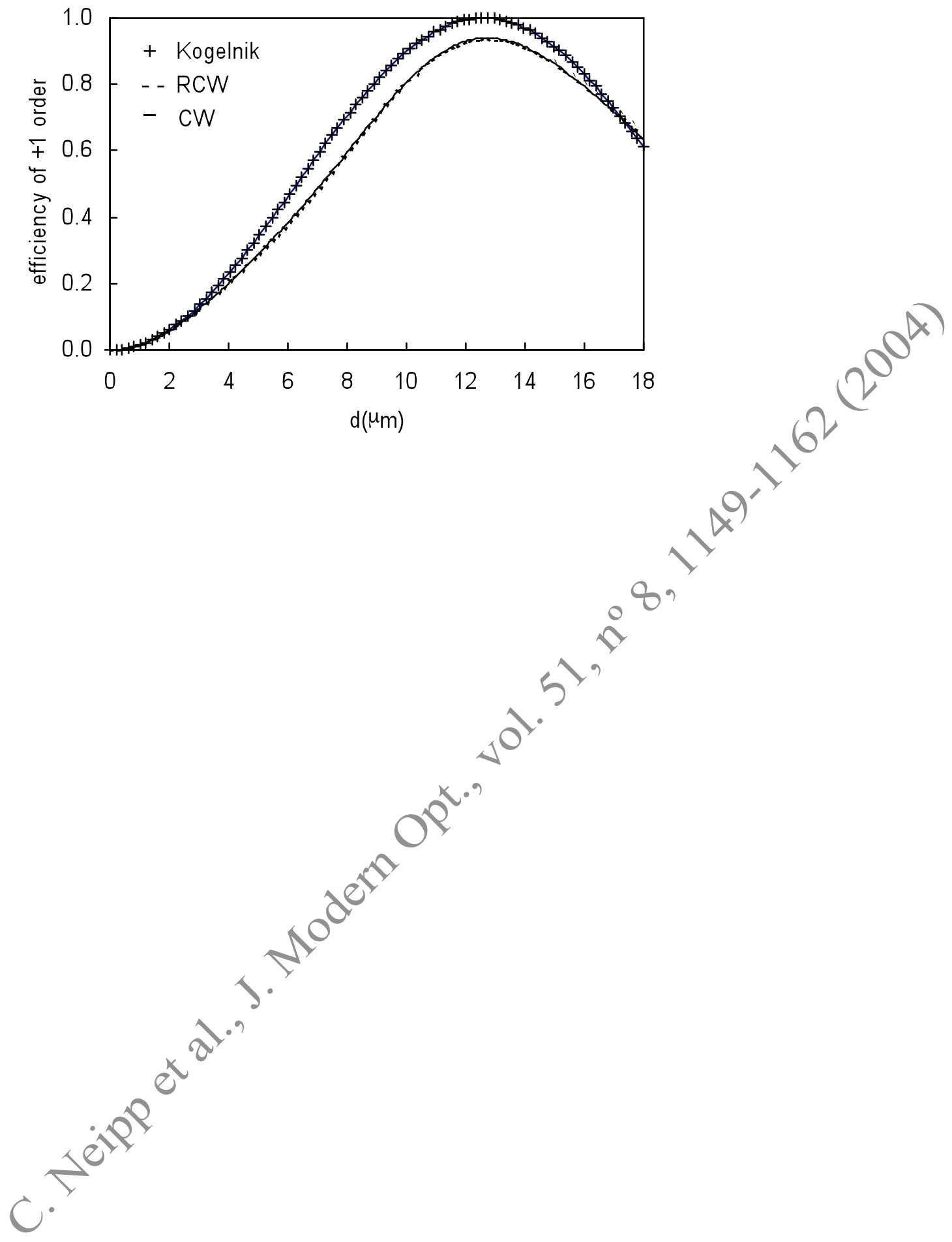

Figure 12 

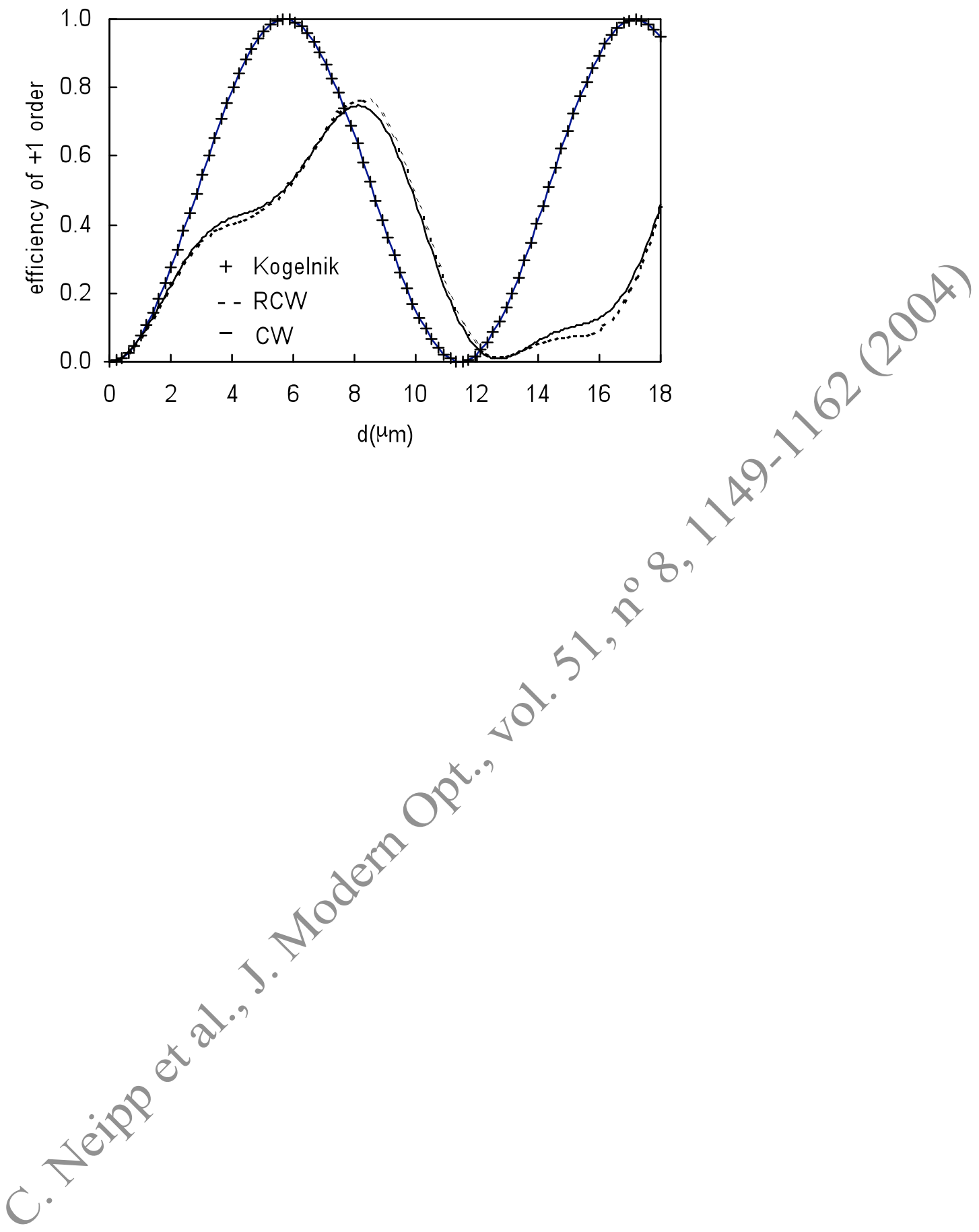

Figure 12 

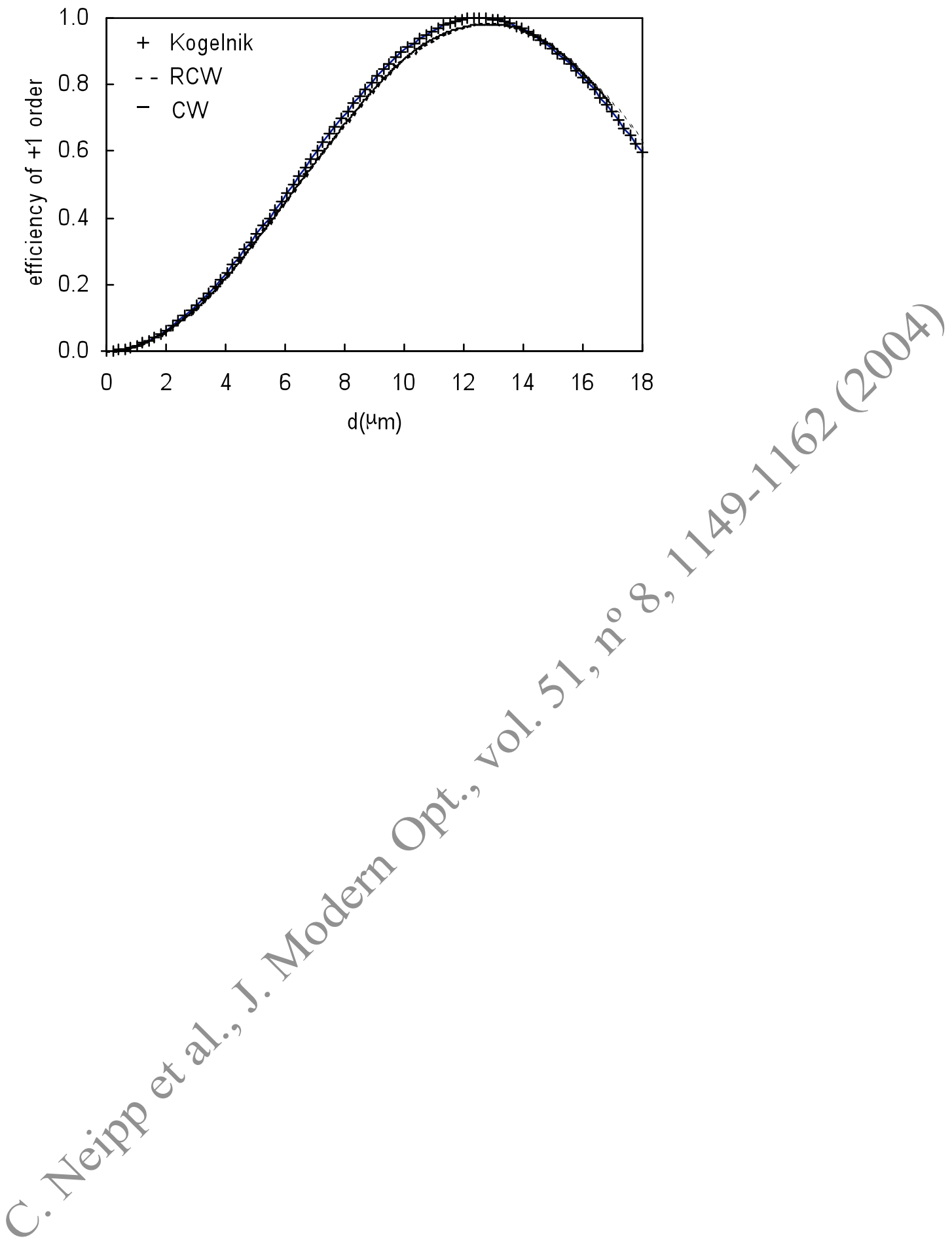

Figure 12 

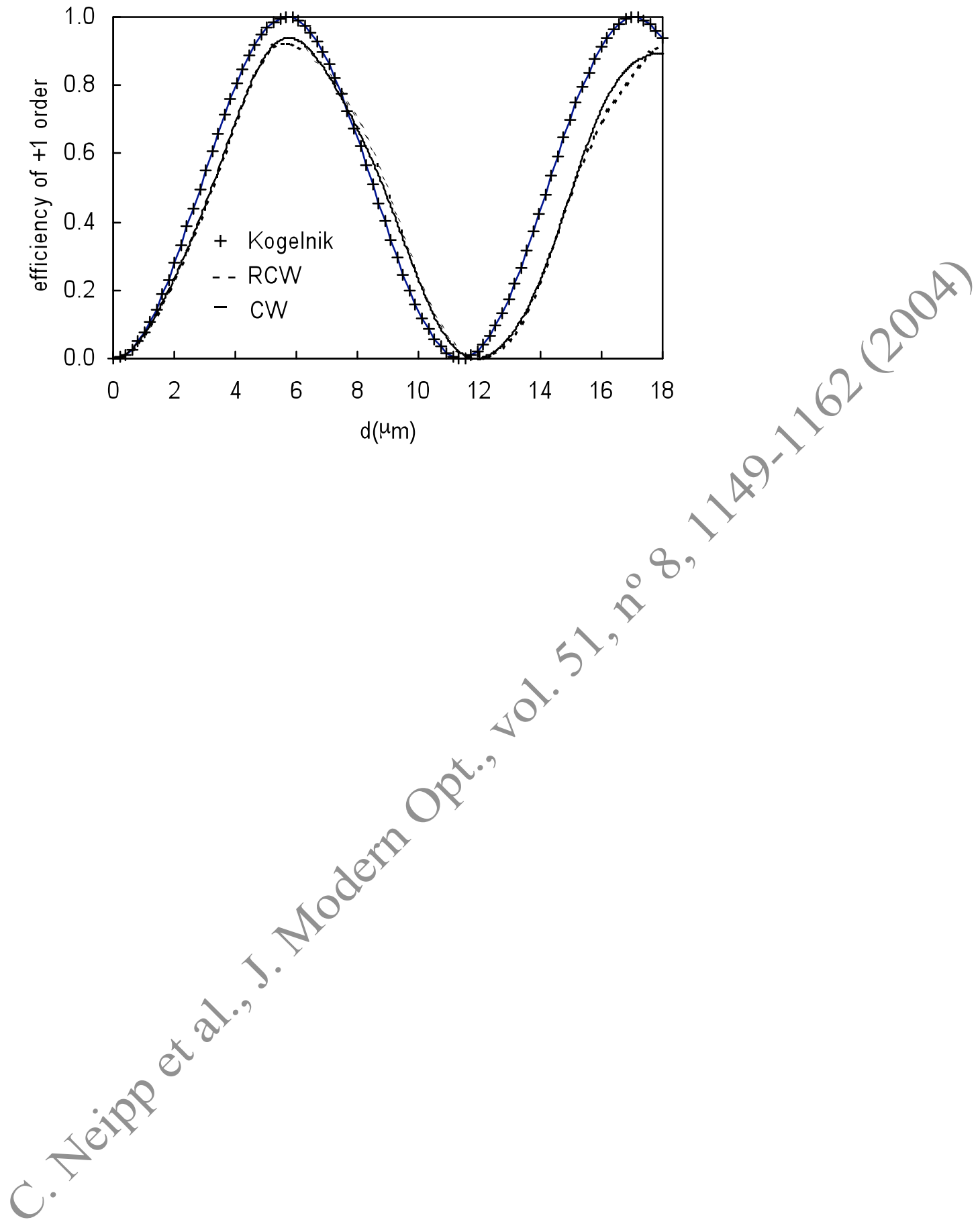

Figure 12 

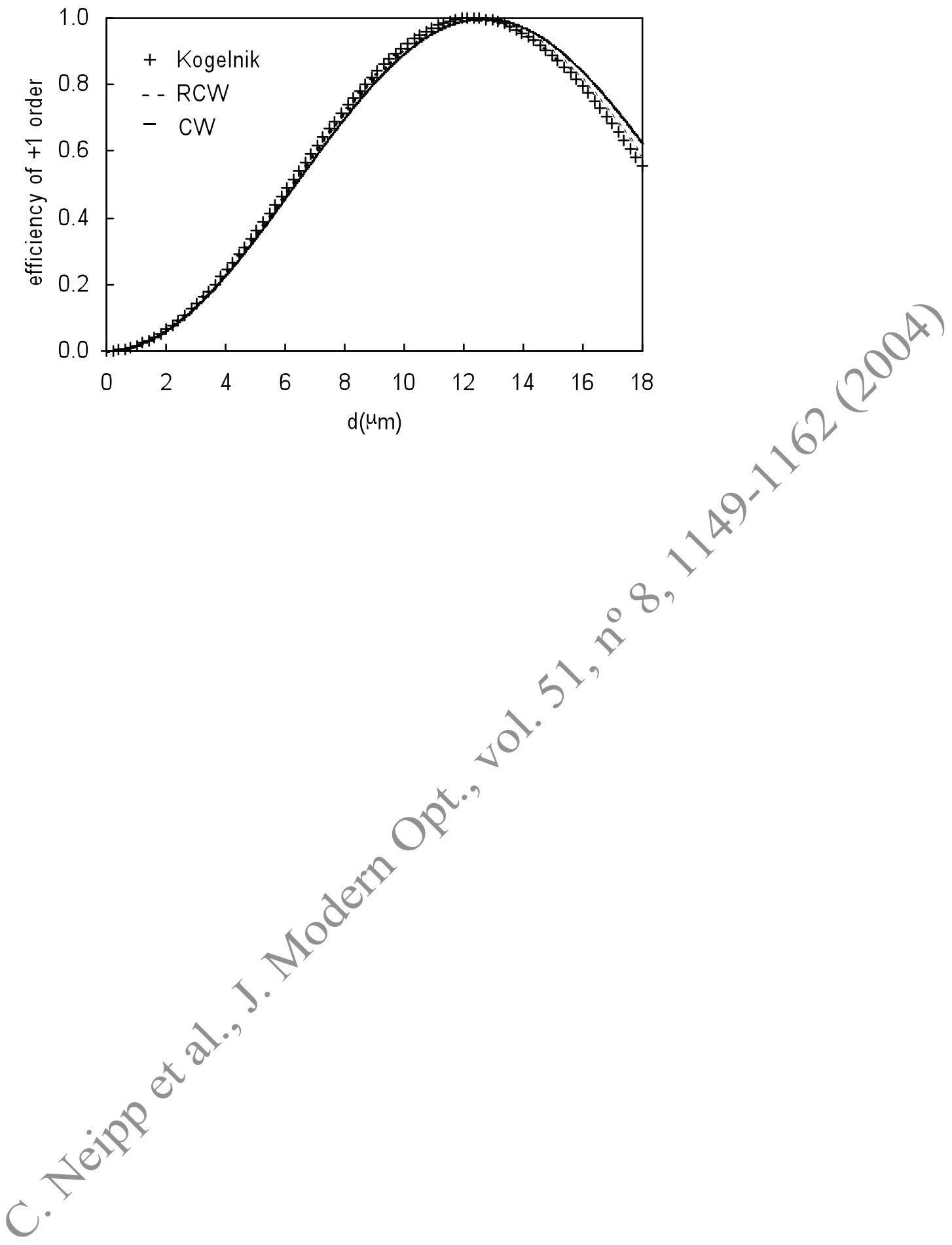

Figure 12 

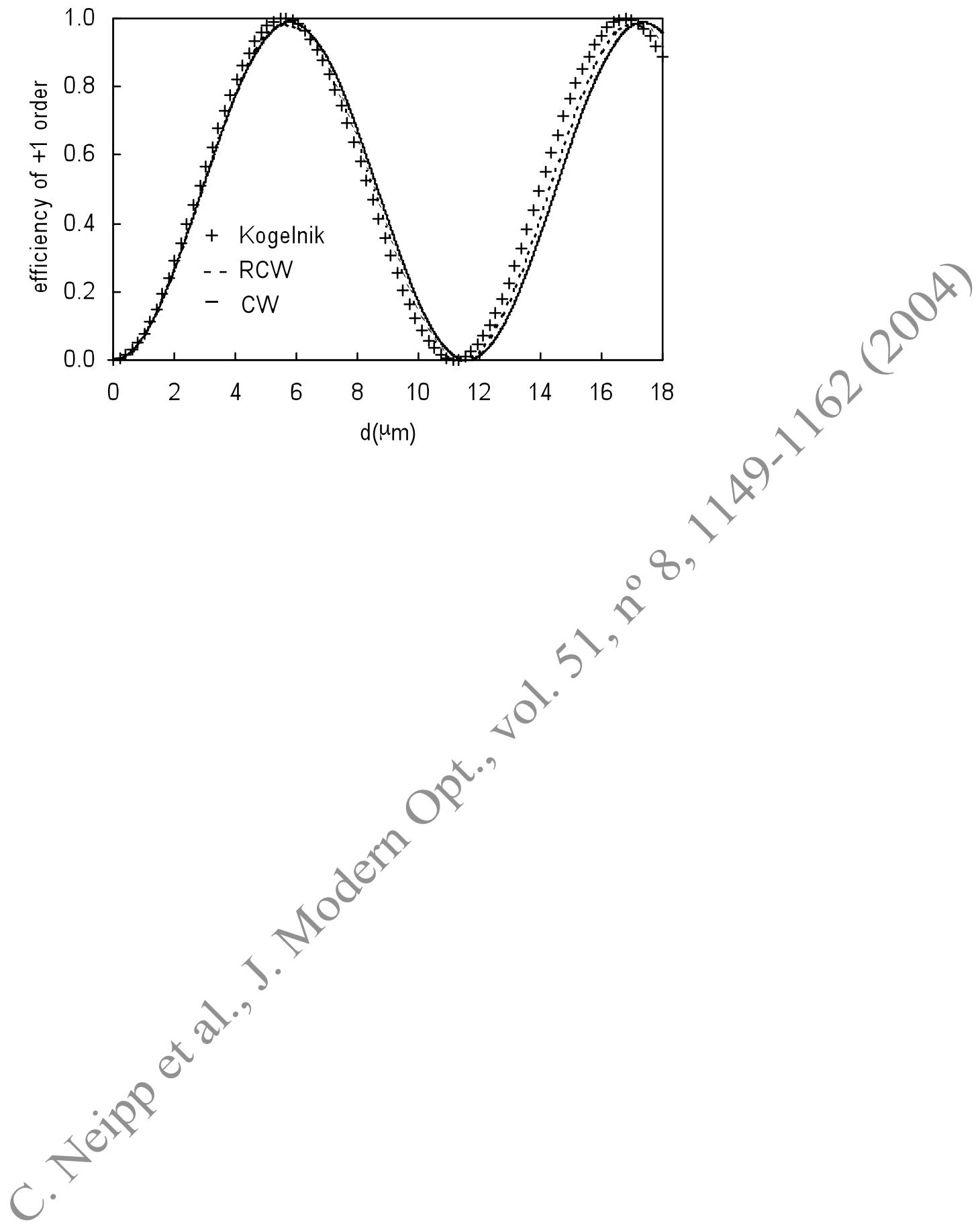

Figure 12 


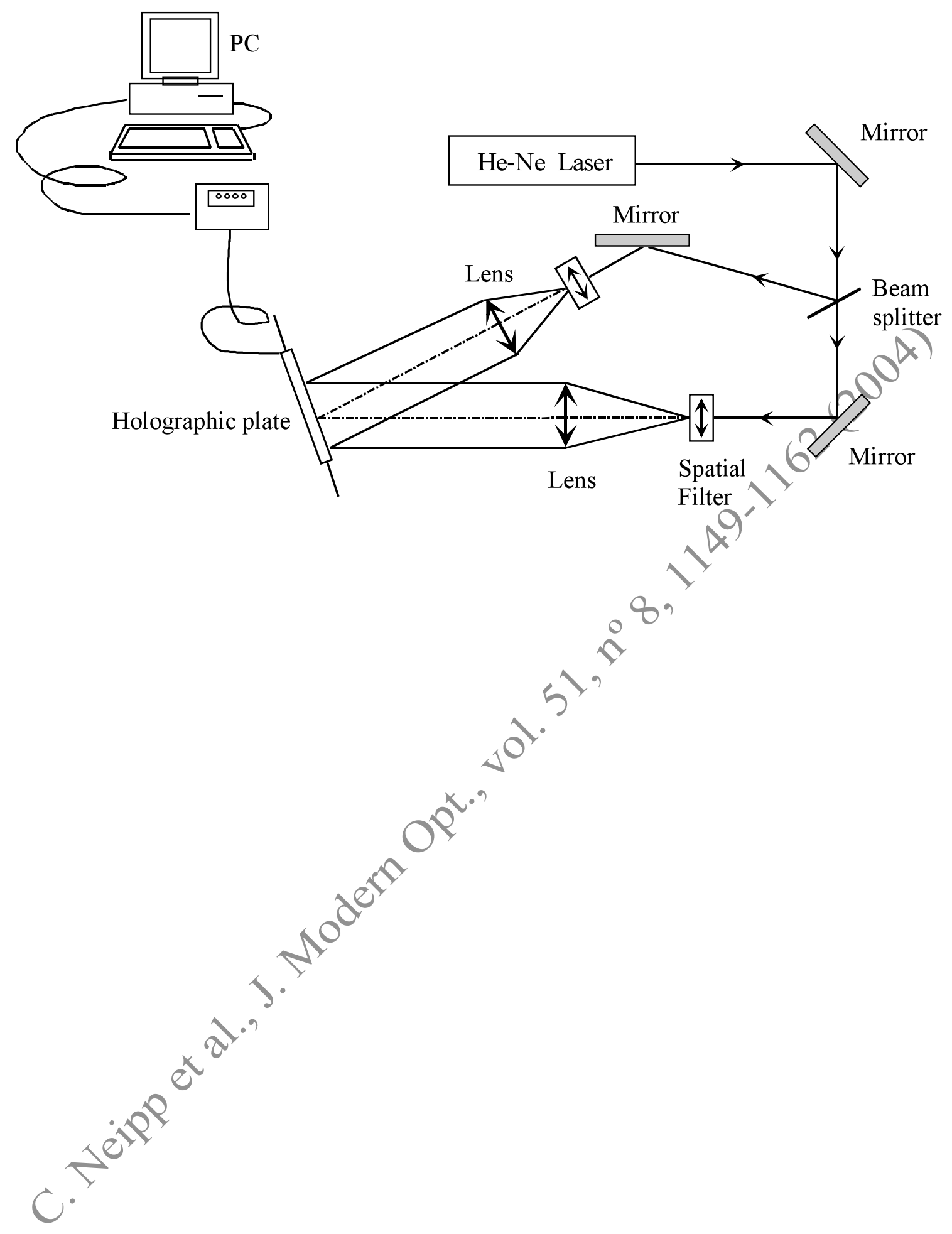

Figure 12 

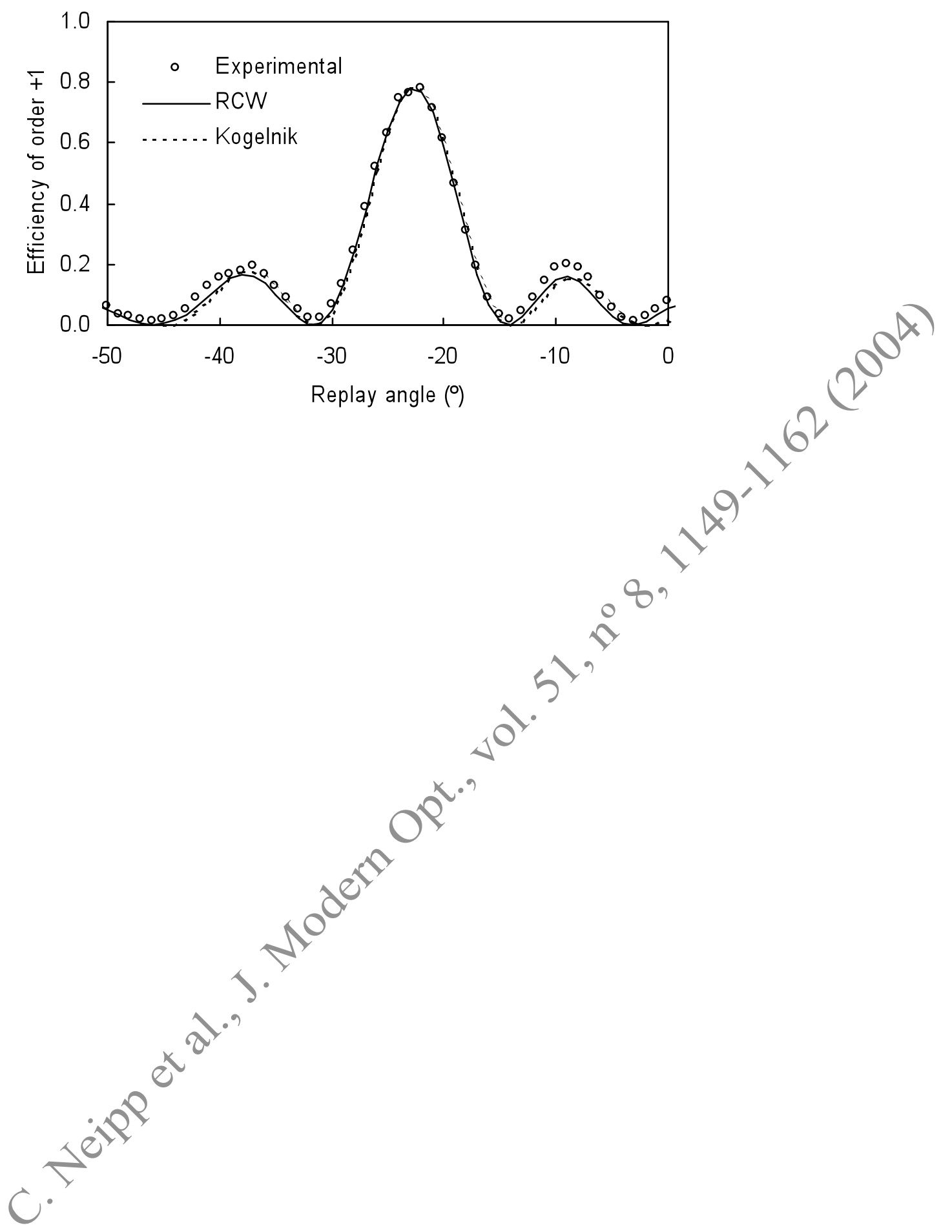

Figure 12 


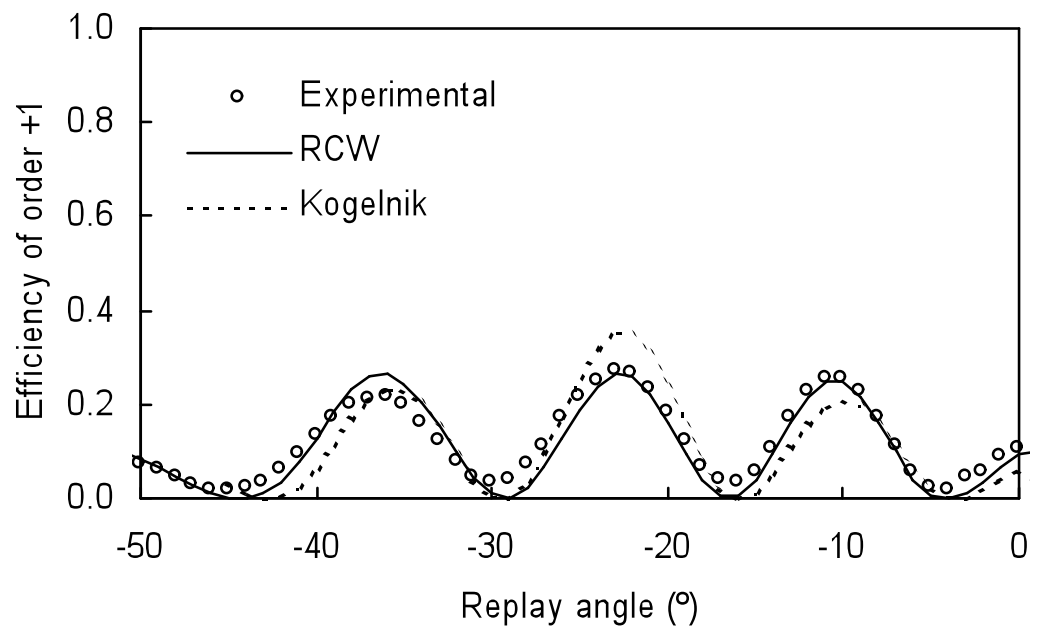




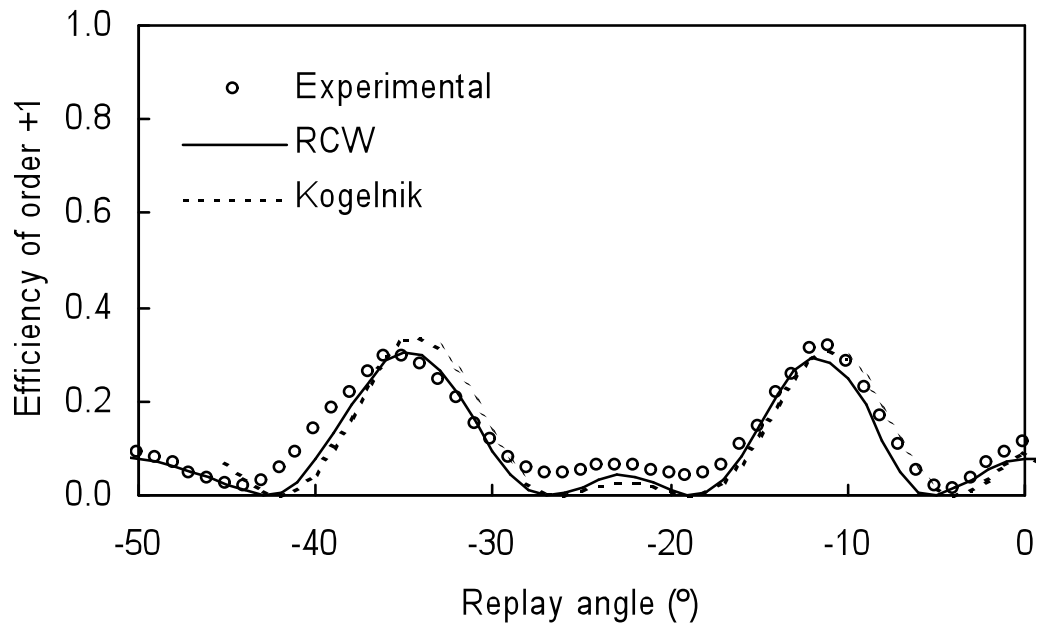

Replay angle $\left({ }^{\circ}\right)$

Figure 12 


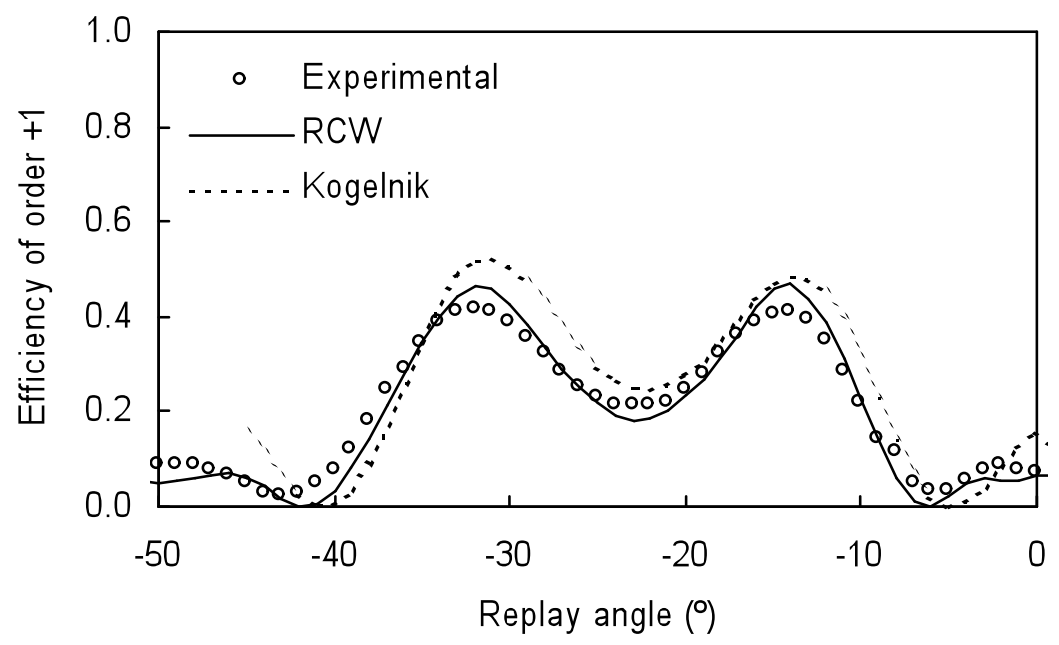

